\title{
SUBPICOSECOND PULSE GENERATION IN A BRANCHED PASSIVELY MODE-LOCKED Nd-GLASS LASER
}

\author{
F. GRAF, J. SCHMIDT and A. PENZKOFER \\ Naturwissenschaftliche Fakultät II - Physik, Universität Regensburg, 8400 Regensburg, Fed. Rep. Germany
}

Received 5 February 1985

\begin{abstract}
A two-branch passively mode-locked Nd-glass laser is applied to generate light pulses down to 0.4 ps duration (average duration $0.9 \mathrm{ps}$ ). The laser action is initiated in a path of high saturable dye transmission and then switched to a branch of low saturable dye transmission for efficient pulse compression. The experimental results are compared with computer simulations.
\end{abstract}

\section{Introduction}

The pulse duration of conventional passively modelocked Nd-phosphate glass lasers (saturable absorber Kodak dye No. 9860) is about 5 to 6 ps. The obtained pulse durations result from pulse broadening in the pre-mode-locking region (natural mode selection) and pulse shortening (saturable absorber) and broadening (natural mode selection, nonlinear losses) in the mode-locking region [1]. Various techniques have been applied to generate shorter pulses in a Ndglass laser oscillator: (i) The natural mode selection in the pre-laser and linear laser region was reduced with Fabry-Perot etalons [2-4]. (ii) The pulse shortening action of the saturable dye was enlarged by introducing linear losses in the rising part of the pulse train with a Pockels cell-polarizer attenuator [1]. (iii) Saturable absorbers with extremely short absorption recovery time were applied in a $\mathrm{z}$-folded oscillator $[5,6]$ (dye No. 5: absorption recovery time $\tau_{\mathrm{D}}=2.7 \mathrm{ps}[7]$, average pulse duration $\Delta t=1.7$ ps [5]; dye No. 3321 : $\tau_{\mathrm{D}}<1 \mathrm{ps}, \Delta t=0.7 \mathrm{ps}$ [6]). Outside the laser oscillator picosecond laser pulses from a Nd-glass laser have been shortened in a multi-pass saturable absorber amplifier system $[8,9]$ and in a regenerative amplifier system [10].

In this paper we describe a two-branch Nd-glass laser oscillator [11] which operates similar to the master-slave oscillator system of [10]: The two branches have the active medium in common. The laser action starts in the branch with high saturable dye transmission (master oscillator) and is switched over to the branch with low saturable dye transmission (slave oscillator) using a Pockels cell pulse selector. Average pulse durations of $0.9 \mathrm{ps}$ and minimum pulse durations down to $0.4 \mathrm{ps}$ have been obtained.

\section{Experimental arrangement}

The experimental setup of the branched laser oscillator is depicted in fig. 1a. The resonator consists of three mirrors M1-M3, three saturable absorber cells DC1-DC3, the active medium AM, the krypton triggered Pockels cell PC1, the thin-film polarizer TFP, and the variable attenuator glass plates GP1, GP2. In the contacted dye cell DC1 the Kodak dye No. 9860 is used. Its small signal single-pass transmission is set to $T_{01}=0.85$. The dye cell DC2 contains the dye No. 5 with a small signal pass transmission of $T_{02}=0.82$. In DC3 dye No. 9860 and No. 5 with small signal single pass dye transmissions between $T_{03}=0.07$ and 0.003 are tested. The thin film polarizer TFP reflects vertically polarized light and transmits horizontally polarized light. The laser action starts between mirrors $\mathrm{M} 1$ and $\mathrm{M} 2$ with vertical polarization since the losses along path $(\mathrm{A}, \mathrm{I})$ are less than the losses along path (A, II). In the rising part of the pulse train photodetector PD2 triggers the krytron system KR1 [12] and a quarter-wave voltage pulse of $12 \mathrm{~ns}$ duration is 


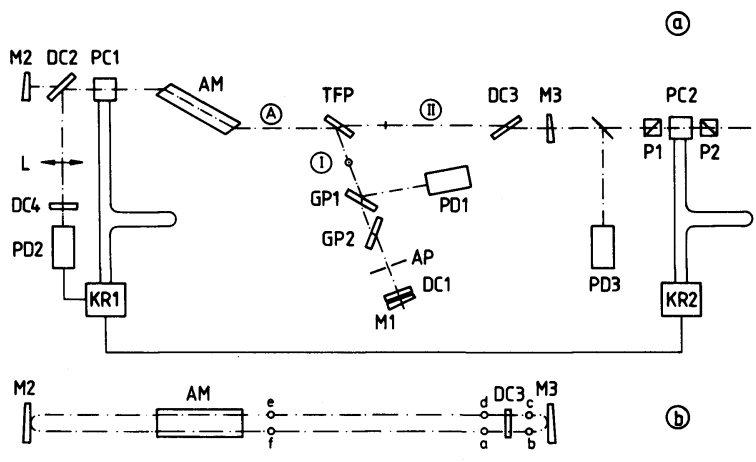

Fig. 1. (a) Experimental setup. M1, plane $100 \%$ mirror, M2, curved $100 \%$ mirror $\left(\rho_{2}=3 \mathrm{~m}\right)$; M3, plane $50 \%$ mirror. DC1 -DC4, saturable absorber cells. AP, aperture $(1.9 \mathrm{~mm}$ hole diameter). GP1, GP2 glass wedges for loss adjustment (one side anti-reflection coated). TFP, thin film polarizer. AM, Nd-phosphate glass rod type Schott LG703. PC1, PC2, Pockels cells. P1, P2, polarizers. KR1, KR2, krytron systems. PD1 -PD3, photodetectors. L, lens $(f=15 \mathrm{~cm})$. (b) Schematic of light path in branch (A, II).

applied to Pockels cell PC1 [path length (A, I): $L_{1} \approx$ $1.7 \mathrm{~m}$; path length $(\mathrm{A}, \mathrm{II}): L_{2} \approx 2.3 \mathrm{~m}$ ]. An accurate adjustment of the trigger threshold is achieved by passing the pulse train reflected at DC2 through a lens $\mathrm{L}(f=15 \mathrm{~cm})$ and a saturable dye cell DC4 (No. 9860, $T_{04}=0.01$ ).

The Pockels cell rotates the polarization of the circulating picosecond pulse by $90^{\circ}$ and the pulse begins to propagate along path (A, II). During each passage through the dye cell DC3 the pulse bleaches partially the dye and it is shortened. Part of the pulse passes through the output mirror M3 (reflectivity $R_{3}=0.5$ ) and forms a train. From this pulse train a single pulse is selected by the krytron triggered Pockels cell PC2. Krytron KR2 is triggered from the synchronisation output of krytron KR1 at a delayed time determined by the length of the connecting cable.

Photodetector PD1 monitors pulse trains along path (A, I). Two typical shapes are shown in fig. 2a. The circles indicate a pulse train which is obtained when the Pockels cell is not operated. The sharp pulse train maximum is caused by self-focusing $[13,14]$ which increases the losses at aperture AP. The vertical lines show a pulse train when the Pockels cell is switched. Photodetector PD3 registers pulse trains along path (A, II). Fig. 2b depicts a typical shape (DC3: No. 9860, $T_{03}=0.003 ;$ M3: plane, $R_{3}=0.5$ ).

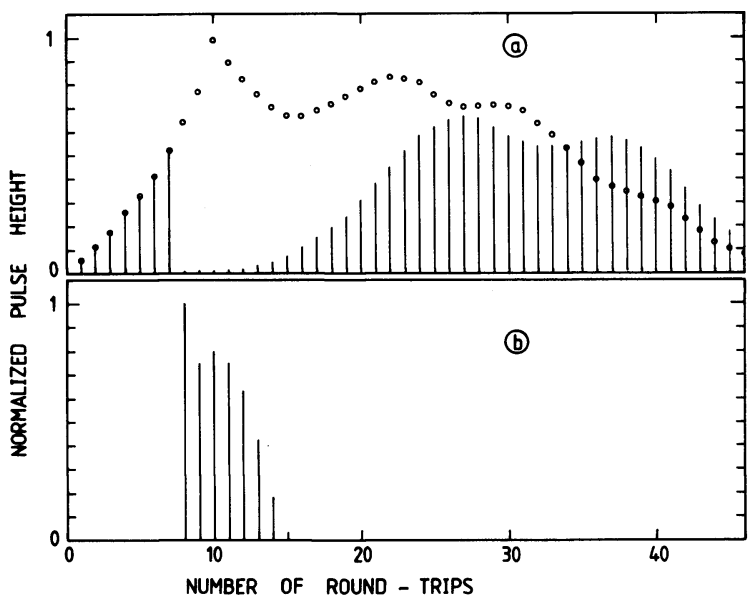

Fig. 2. (a) Pulse trains in branch (A, I). Circles, without operation of Pockels cell PC1. Solid lines, with pulse switching. (b) Switched pulse in branch (A, II). DC3 contains dye No. 9860 with $T_{03}=0.003$.

At the switching moment the losses along both paths (A, I) and (A, II) have to be approximately equal in order to achieve optimum pulse shortening. This condition is fulfilled by loss adjustment in path $(\mathrm{A}, \mathrm{I})$ by proper tilting the glass plates GP1 and GP2.

The spectra of the selected single picosecond pulses behind PC2 are measured with a $25 \mathrm{~cm}$-grating spectrometer. The pulse durations are measured after passage of the selected single pulses through a Nd-phosphate glass amplifier. The two-photon fluorescence technique [15] is applied (absorber $2.5 \times 10^{-3}$ molar rhodamine $6 \mathrm{G}$ in ethanol).

\section{Results}

The insertion of Pockels cell PC1 into the resonator elongates the pulses slightly. The pulse durations obtained along path $(\mathrm{A}, \mathrm{I})$ without dye No. 5 in DC2 ranged between 5 to 7 ps (without Pockels cell $\Delta t \approx 5-6 \mathrm{ps})$. Dye No. 5 in DC2 $\left(T_{0}=0.82\right)$ extends the pulse shortening period along the pulse train (higher saturation intensity) and pulses with $\Delta t=$ $(4.0 \pm 0.4)$ ps duration (FWHM) and $\Delta \widetilde{\nu}=(25 \pm 5)$ $\mathrm{cm}^{-1}$ spectral width (FWHM) are generated at the switching position to path (A, II).

The pulse shortening in path (A, II) is depicted in fig. 3. Data points (open circles for dye No. 9860 , 


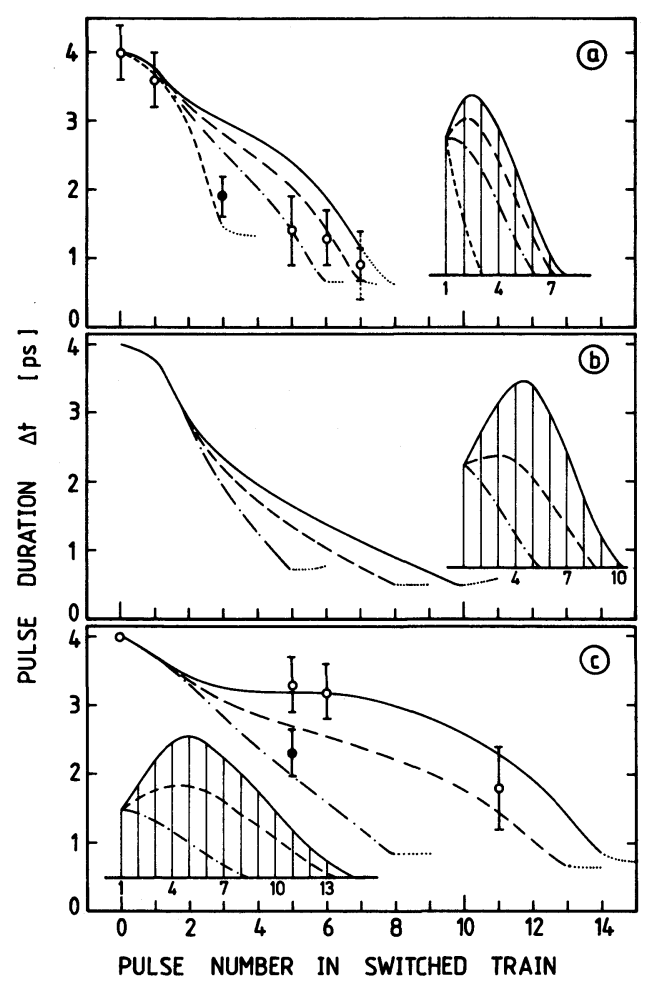

Fig. 3. Pulse shortening in branch (A, II). Open circles, dye No. 9860 ; closed circles, dye No. 5 . Short-broken curve in (a) dye No. 5 , all other curves belong to dye No. 9860. (a) $T_{03}=$ 0.003 , two-photon absorption cross-section $\alpha^{(2)}=4 \times 10^{-12}$ $\mathrm{cm} / \mathrm{W}$, rod length $l_{\mathrm{R}}=13 \mathrm{~cm}$. Input peak intensity $I_{0 \mathrm{a}}(j=1)$ $=10^{10} \mathrm{~W} / \mathrm{cm}^{2}$ and $2.5 \times 10^{10} \mathrm{~W} / \mathrm{cm}^{2}$ for No. 9860 and No. 5 , respectively. Solid bars indicate single shot standard deviation; the dashed bar includes all measured durations. (b) $T_{03}=$ $0.003, \alpha^{(2)}=0, I_{0 \mathrm{a}}(j=1)=10^{10} \mathrm{~W} / \mathrm{cm}^{2}$. Dye No. 9860 . (c) $T_{03}=0.07, \alpha^{(2)}=4 \times 10^{-12} \mathrm{~cm} / \mathrm{W}, l_{\mathrm{R}}=13 \mathrm{~cm}, I_{0 \mathrm{a}}(j=1)$ $=5 \times 10^{9} \mathrm{~W} / \mathrm{cm}^{2}$. Insets represent pulse energy densities $\int I_{\mathrm{b}}\left(t^{\prime}, j\right) \mathrm{d} t^{\prime}$ (pulse train at position $\mathrm{b}$ of fig. $1 \mathrm{~b}$ ). Solid curves: $g=1.2$; dashed curves: $g=1$; dash-dotted curves: $g=0.8$. Short-broken curve: dye No. 5. Dotted parts of curves: pulse energy too low for detection. Focusing factor $f=0.5$ (eq. (9)). Dye parameters, see fig. 4.

closed circles for No. 5) are shown for $T_{03}=0.07$ (fig. 3c) and 0.003 (fig. 3a). For $T_{03}=0.003$ and dye No. 9860 the pulse durations were reduced from an initial value of $4.0 \mathrm{ps}$ to an average value of $0.9 \mathrm{ps}$ after seven round-trips. The spectral widths increased only slightly from $\Delta \tilde{\nu} \approx 25 \mathrm{~cm}^{-1}$ to $\Delta \tilde{\nu} \approx 40 \mathrm{~cm}^{-1}$. The spectral shapes were rather smooth. The shortest pulse duration obtained was $\Delta t=0.4 \mathrm{ps}$.

\section{Discussion}

The pulse propagation along path $(\mathrm{A}, \mathrm{II})$ is shown schematically in fig. $1 \mathrm{~b}$ (cell DC2 is neglected). The saturable dye in DC3 is described by a four-level system as shown by the inset in fig. $4 \mathrm{~b}$. The light passage through the dye cell is governed by eqs. (1)-(5) [17, 18]:

$$
\begin{aligned}
& \partial N_{1} / \partial t^{\prime}=-(I / h \nu) \sigma_{\mathrm{D}}\left(N_{1}-N_{2}\right)+\left(1 / \tau_{\mathrm{D}}\right)\left(N_{2}+N_{3}\right), \\
& \partial N_{2} / \partial t^{\prime}=(I / h v) \sigma_{\mathrm{D}}\left(N_{1}-N_{2}\right) \\
& \quad-(I / h \nu) \sigma_{\mathrm{ex}} N_{2}-\left(1 / \tau_{\mathrm{FC}}\right) N_{2}-\left(1 / \tau_{\mathrm{D}}\right) N_{2} \\
& \partial N_{3} / \partial t^{\prime}=\left(1 / \tau_{\mathrm{FC}}\right) N_{2} \\
& \quad-(I / h \nu) \sigma_{\mathrm{ex}}\left(N_{3}-N_{4}\right)-N_{3} / \tau_{\mathrm{D}}+N_{4} / \tau_{\mathrm{ex}} \\
& \partial N_{4} / \partial t^{\prime}=(I / h v) \sigma_{\mathrm{ex}}\left(N_{2}+N_{3}-N_{4}\right)-N_{4} / \tau_{\mathrm{ex}} \\
& \partial I / \partial z=-I \sigma_{\mathrm{D}}\left(N_{1}-N_{2}\right)-I \sigma_{\mathrm{ex}}\left(N_{2}+N_{3}-N_{4}\right)
\end{aligned}
$$

The coordinate transformation $t^{\prime}=t-\left(n / c_{0}\right) z$ is used ( $n$ refractive index, $c_{0}$ vacuum light velocity). $\sigma_{\mathrm{D}}$ and $\sigma_{\mathrm{ex}}$ are the ground state and excited state absorption cross-section, respectively. $\tau_{D}$ is the $S_{1}$-state lifetime. $\tau_{\mathrm{FC}}$ is the Franck-Condon relaxation time within the $\mathrm{S}_{1}$-band. $\tau_{\mathrm{ex}}$ describes the relaxation of higher excited singlet states. $\nu$ is the laser frequency. The initial conditions for the population densities $N_{1}$ to $N_{4}$ before light pulse passage are $N_{1}\left(t^{\prime}=-\infty, z\right)=N_{0}=$ $-\ln \left(T_{03}\right) / \sigma_{\mathrm{D}} l_{\mathrm{D}}, N_{2}\left(t^{\prime}=-\infty, z\right)=N_{3}\left(t^{\prime}=-\infty, z\right)=$ $N_{4}\left(t^{\prime}=-\infty, z\right)=0 . l_{\mathrm{D}}$ is the length of sample DC3. The initial pulse shape of the switched pulses at position a (fig. 1b) before their first circulation in path (A, II) is assumed to be gaussian, i.e. $I_{\mathrm{a}}\left(t^{\prime}, j=1\right)=$ $I_{0 \mathrm{a}}(j=1) \exp \left(-t^{\prime 2} / t_{0}^{2}\right)\left(t_{0}=\Delta t /\left\{2[\ln (2)]^{1 / 2}\right\}, \Delta t=\right.$ $4.0 \mathrm{ps})$. The intensity transmission through the dye is $T_{\mathrm{I}}=I_{\text {out }}\left(t^{\prime}\right) / I_{\text {in }}\left(t^{\prime}\right)$. The power transmission is $T_{\mathrm{p}}=$ $\int I_{\text {out }}\left(t^{\prime}\right) \mathrm{d} t^{\prime} / \int I_{\text {in }}\left(t^{\prime}\right) \mathrm{d} t^{\prime}$. The equation system (1)-(5) neglects absorption anisotropy $[17,18]$ and spatial pulse shape. The power transmission curves without absorption anisotropy are nearly equal to the energy transmission curves

$$
\begin{aligned}
T_{\mathrm{E}} & =\iint I_{\text {out }}\left(t^{\prime}, r\right) 2 \pi r \mathrm{~d} r \mathrm{~d} t^{\prime} \\
& \times\left(\iint I_{\text {in }}\left(t^{\prime}, r\right) 2 \pi r \mathrm{~d} r \mathrm{~d} t^{\prime}\right)^{-1}
\end{aligned}
$$


with absorption anisotropy [16].

The pulse circulation in resonator (A, II) is described by equations (6)-(12) for the intensities (see fig. 1b)

$I_{\mathrm{b}}\left(t^{\prime}, j\right)=I_{\mathrm{a}}\left(t^{\prime}, j\right) T_{\mathrm{I}}\left(I_{\mathrm{a}}\right)$,

$I_{\mathrm{c}}\left(t^{\prime}, j\right)=I_{\mathrm{b}}\left(t^{\prime}, j\right) R_{3}$,

$I_{\mathrm{d}}\left(t^{\prime}, j\right)=I_{\mathrm{c}}\left(t^{\prime}, j\right) T_{\mathrm{I}}\left(I_{\mathrm{c}}\right)$,

$I_{\mathrm{e}}\left(t^{\prime}, j\right)=I_{\mathrm{d}}\left(t^{\prime}, j\right) f$

$I_{\mathrm{f}}\left(t^{\prime}, j\right)=I_{\mathrm{e}}\left(t^{\prime}, j\right) G_{j} T_{\mathrm{TPA}}\left(t^{\prime}, j\right)$,

$I_{\mathrm{f}}\left(t^{\prime}, j+1\right)=I_{\mathrm{f}}\left(t^{\prime}\left[1+\left(\tau_{\text {res }} / \Delta t\right)^{2}\right]^{1 / 2}, j\right)$,

$I_{\mathrm{a}}\left(t^{\prime}, j+1\right)=I_{\mathrm{f}}\left(t^{\prime}, j+1\right) / f$.

The factor $f$ takes care of the intensity changes between active medium and dye cell due to resonator geometry. $G_{j}$ represents the pulse amplification in the active medium. The initial gain at the first passage is

$$
\begin{aligned}
G_{1} & =\exp \left[2 l_{\mathrm{A}} \sigma_{\mathrm{E}} N_{\mathrm{A}}(j=1)\right] \\
& =\frac{g\left[1+\alpha^{(2)} 2 l_{\mathrm{R}} I_{0 \mathrm{a}}(j=1) f\right]}{T_{\mathrm{p}}\left(I_{\mathrm{a}}, j=1\right) R_{3} T_{\mathrm{p}}\left(I_{\mathrm{c}}, j=1\right)},
\end{aligned}
$$

$l_{\mathrm{A}}$ is the pumped length of the active medium, $\sigma_{\mathrm{E}}$ is the effective stimulated emission cross-section and $N_{\mathrm{A}}$ the upper laser level population density. $g$ represents the net gain of the peak pulse intensity for the first round-trip along path (A, II). The factor in brackets behind $g$ compensates two-photon absorption losses. The gain reduces due to depletion of inversion by stimulated emission

$N_{\mathrm{A}}(j+1)=N_{\mathrm{A}}(j)-\left(G_{j}-1\right) \int I_{\mathrm{e}}\left(t^{\prime}\right) \mathrm{d} t^{\prime} /\left(h \nu 2 l_{\mathrm{A}}\right)$,

according to

$G_{j+1}=G_{j} \exp \left(-\sigma_{\mathrm{E}}\left(G_{j}-1\right) \int I_{\mathrm{e}}\left(t^{\prime}\right) \mathrm{d} t^{\prime} / h \nu\right)$.

$T_{\text {TPA }}$ takes care of the two-photon absorption in the active medium [19]. It is approximated by

$T_{\mathrm{TPA}}\left(t^{\prime}, j\right)=\left[1+l_{\mathrm{R}} \alpha^{(2)} I_{\mathrm{e}}\left(t^{\prime}, j\right) G_{j}\right]^{-1}$,

$l_{R}$ is the rod length of the active medium. $\alpha^{(2)}$ is the two-photon absorption coefficient.

The finite band width of the gain profile of the active medium leads to a finite response time of the active medium which broadens the pulse duration. We take into consideration this broadening by eq. (11) [1]. The response time is estimated to be $\tau_{\text {res }} \approx$ $0.5 / \Delta \nu_{\mathrm{A}}$ where $\Delta \nu_{\mathrm{A}}$ is the fluorescence linewidth of the laser transition.

Fig. $4 \mathrm{~b}$ shows the power transmission for the first double passage through dye cell DC3 (path $a-b-c-d$ ). The curves are calculated for dyes No. 9860 and No. 5 with $T_{03}=0.003$ and 0.07 . Fig. $4 \mathrm{a}$ depicts the corresponding pulse shortening in the first double passage through the dye. At optimum pulse shortening the losses are too high. For dye No. 9860 and $T_{03}=0.003$ the input peak intensity has to be $I_{0 \mathrm{a}}(j=1) \geqslant 8 \times 10^{9}$ $\mathrm{W} / \mathrm{cm}^{2}$ to achieve several round-trips. For dye No. 5 and $T_{03}=0.003$ the input peak intensity has to be

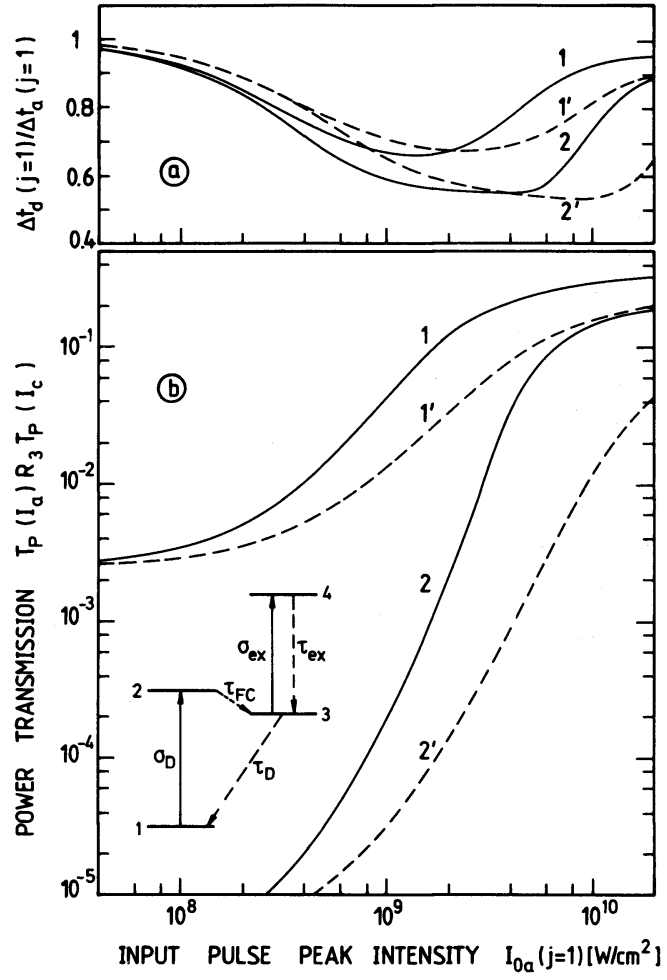

Fig. 4. Pulse shortening (a) and power transmission of switched pulse $(j=1)$ along passage from a to $\mathrm{d}$ of fig. $1 \mathrm{~b}$. Solid curves, dye No. $9860\left(\sigma_{\mathrm{D}}=3.7 \times 10^{-16} \mathrm{~cm}^{2}, \sigma_{\mathrm{ex}}=\right.$ $\left.2.5 \times 10^{-17} \mathrm{~cm}^{2}[21], \tau_{\mathrm{FC}}=0.7 \mathrm{ps}, \tau_{\mathrm{ex}}=0.1 \mathrm{ps}\right)$; broken curves, dye No. $5\left(\sigma_{\mathrm{D}}=3 \times 10^{-16} \mathrm{~cm}^{2}, \sigma_{\mathrm{ex}}=5 \times 10^{-17}\right.$ $\left.\mathrm{cm}^{2}[16], \tau_{\mathrm{FC}}=0.7 \mathrm{ps}, \tau_{\mathrm{ex}}=0.1 \mathrm{ps}\right)$. Input pulse: temporal gaussian shape, duration $\Delta t_{\mathrm{a}}(j=1)=4$ ps. Curves $1,1^{\prime}$, $T_{03}=0.07$; curves $2,2^{\prime}, T_{03}=0.003$. Inset: Level scheme of saturable dye. 
$I_{0 \mathrm{a}}(j=1) \geqslant 2 \times 10^{10} \mathrm{~W} / \mathrm{cm}^{2}$ to achieve three transits (a to b) through DC3. The higher excited state absorption of dye No. $5\left(\sigma_{\mathrm{ex}}=5 \times 10^{-17} \mathrm{~cm}^{2},[16]\right.$ and this work) makes this dye less suitable for short pulse generation in path (A, II) than dye No. 9860 $\left(\sigma_{\mathrm{ex}}=2.5 \times 10^{-17} \mathrm{~cm}^{2}[21]\right)$ despite its shorter absorption recovery time. For low initial dye transmission the number of transits depends very sensitively on the excited state absorption.

Fig. 3 presents some calculated curves of pulse duration versus number of passages in path (A, II) for $T_{03}=0.003$ (fig. 3a and 3b) and $T_{03}=0.07$ (fig. 3c). The curves in fig. 3a and 3c are calculated for $\alpha^{(2)}=$ $4 \times 10^{-1.2} \mathrm{~cm} / \mathrm{W}$ while the curves of fig. $3 \mathrm{~b}$ are calculated without two-photon absorption. The curves are calculated for gain values $g$ (eq. (13)) around the optimum shortening region. They agree well with the measured data points. All curves belong to dye No. 9860 except the short-broken curve of fig. 3a which belongs to dye No. 5. For this curve the gain at the switching position was set to the experimentally obtainable maximum value of $G_{1}=13.5$.

The insets in fig. 3 represent the corresponding pulse energies (pulse trains as detected by PD3 of fig. 1a). They are similar to the experimental pulse trains (typical case shown in fig. 2b) except the downward step between the first and second pulse of the experimental trains. This energy reduction between the first and the second pulse is caused by changes of the spatial beam profile due to the soft-aperture action [20] of the dye in DC3 and due to resonator changes from path $(A, I)$ to path $(A, I I)$. In the calculations a rectangular spatial beam profile is assumed.

\section{Conclusions}

With the described branched Nd-phosphate glass laser resonator intense subpicosecond light pulses have been generated. They may be applied to subpicosecond time resolved spectroscopy and nonlinear optical studies.

\section{Acknowledgement}

The authors thank Th. Ascherl for technical assistance and the Rechenzentrum of the University for disposal of computer time.

\section{References}

[1] F. Graf, G. Pleininger and A. Penzkofer, Appl. Phys. B34 (1984) 123.

[2] D. von der Linde and K.F. Rodgers, Optics Comm. 8 (1973) 91.

[3] E.M. Gordeev, P.G. Kryukov, Yu.A. Matveets, B.M. Stepanov, S.D. Fanchenko, S.V. Cheaklin and A.V. Starkov, Sov. Phys. J. Quant. Electron. 5 (1975) 129.

[4] F. Graf, C. Löw and A. Penzkofer, Optics Comm. 47 (1983) 329.

[5] C. Kolmeder and W. Zinth, Appl. Phys. 24 (1981) 341.

[6] B. Bareika, A. Priskarskas, V. Sinkyarichyus and V. Sirutaikis, Sov. J. Quant. Electron. 14 (1984) 407.

[7] B. Kopainsky, W. Kaiser and K.H. Drexhage, Optics Comm. 32 (1980) 451.

[8] A. Penzkofer, D. von der Linde, A. Laubereau and W. Kaiser, Appl. Phys. Lett. 20 (1972) 351.

[9] A. Penzkofer, Opto-Electron. 6 (1974) 87.

[10] A. Penzkofer, F. Härtinger and J. Wiedmann, Appl. Phys. B26 (1981) 239.

[11] B.P. Boczar and M.R. Topp, Appl. Optics 22 (1983) 1611.

[12] J. Biebl and A. Penzkofer, J. Phys. E: Sci. Instrum. 13 (1980) 1328.

[13] R.C. Eckardt, IEEE J. Quant. Electron. QE-10 (1974) 48.

[14] A.N. Zherikhin, P.G. Kryukov, Yu.A. Matveets and S.W. Chekalin, Sov. J. Quant. Electron. 4 (1974) 249.

[15] J.A. Giordmaine, P.M. Rentzepis, S.L. Shapiro and K.W. Wecht, Appl. Phys. Letters 11 (1967) 216.

[16] F. Graf and A. Penzkofer, Optics Comm. 51 (1984) 111.

[17] A. Penzkofer and W. Falkenstein, Opt. Quant. Electr. 10 (1978) 399.

[18] A. Penzkofer and W. Blau, Opt. Quant. Electr. 15 (1983) 325.

[19] A. Penzkofer and W. Kaiser, Appl. Phys. Lett. 21 (1972) 427.

[20] A. Penzkofer and F. Fröhlich, Optics Comm. 28 (1979) 197.

[21] F. Graf and A. Penzkofer, Opt. Quant. Electron. 17 (1985) in press. 